\title{
Influence of boundary conditions on the stop band effect in finite locally resonant metamaterial beams
}

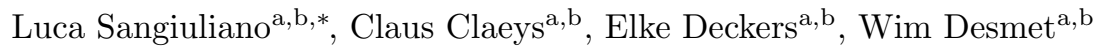 \\ ${ }^{a}$ Department of Mechanical Engineering, KU Leuven, Belgium \\ ${ }^{b} D M M S$ lab, Flanders Make
}

\begin{abstract}
This paper investigates the influence of the boundary conditions on the stop band effect in finite locally resonant metamaterial beams. In a first step, a Timoshenko beam with tuned vibration absorbers is used to describe a metamaterial beam. The influence of different boundary conditions for beams with different lengths as well as of tuned vibration absorbers with different amounts of added mass or different tuned frequencies is assessed numerically. It is found that edge modes are present inside the stop band frequency region predicted by the unit cell analysis and these edge modes reduce the level of vibration attenuation achieved. Three solutions are analysed to reduce the effect of the edge modes: (i) varying the tuned frequency, (ii) the number or (iii) the positions of the individual tuned vibration absorbers added to the host structure. In a second step, the results of the numerical investigation are experimentally validated: the experimental results are in good agreement with the numerical results.
\end{abstract}

Keywords: Locally resonant metamaterials, Boundary conditions, Stop band, Edge modes

\section{Introduction}

In the last decades, stringent environmental regulations and financial requirements have influenced the 3 design of vibro-acoustic treatments, especially in the aviation and automotive sector. The awareness of the 4 effect of noise pollution on human health [1] leads to a demand for enhanced Noise, Vibration, and Harshness (NVH) solutions. At the same time, lightweight design is required to reduce $\mathrm{CO}_{2}$ emissions [2]. Considering

that the NVH performance that a partition can achieve scales with its mass [3], combining lightweight design with enhanced NVH treatments is a challenge.

Among novel NVH solutions, special attention is given to metamaterials. These are materials engineered from conventional materials to exhibit stop band behaviour. Stop bands are frequency range in which free wave propagation is hindered [4] and in which superior noise and vibration insulation properties can be achieved, even beating the mass law. Depending on the governing mechanisms to create stop band

*Corresponding author: luca.sangiuliano@kuleuven.be 
behaviour, metamaterials can be divided according to the class of phononic crystals (PCs) or locally resonant metamaterials (LRMs) [5]. In PCs, stop bands rely on Bragg scattering and hence, on the periodicity in the lattice $[6,7,8]$. In LRMs, the formation of stop bands is due to Fano-type interference [9]. This can be achieved through the addition of small resonant systems to an elastic host structure on a scale smaller than the wavelength of the wave to be affected, hence in a subwavelength scale $[10,11,12]$. As a result, LRMs do not require periodicity, as is the case for PCs, making them suitable for applications that demand compact and lightweight design with enhanced NVH properties in the-hard-to-address frequency range below $1 \mathrm{kHz}$. For this reason, LRMs are further investigated in this work.

Several metamaterial concepts have been investigated numerically and experimentally for different applications [13]: from 1D systems $[14,15,16,17]$ to more complex 2D and 3D lattice structures [18, 19, 20]. Independently of the degrees of complexity of the metamaterial system, stop bands in metamaterials are commonly analysed using the unit cell (UC) modelling approach. By modelling the smallest representative portion of the infinite periodic structure, the UC, and by applying periodic Bloch-Floquet boundary conditions (BCs) [4], the stop band frequencies can be predicted. Depending on the type of UC modelling applied, also the amount of attenuation in the stop band can be assessed [21, 22].

In practice, however, structures are of finite dimensions and their dynamic behaviour is influenced by their dimensions and the applied BCs. As a result, the stop band predicted by UC modelling in infinite periodic metamaterial may not be preserved in its finite counterpart. In the literature, multiple examples can be found of modes with local behaviour occurring in the predicted stop band frequency region $[23,24,25,26,27]$. These modes can impair the designed stop band performance by reducing the vibration attenuation achieved. However, they can be controlled through an ad-hoc design of the finite metamaterial system. In PCs, this can be achieved by perturbing the design of the existing periodic structure with different sizes or material properties [26, 28]. In LRMs, it can be done by varying the relative position between resonant elements [27].

Given the variety of configurations of finite LRMs that can be designed to tackle different industrial NVH problems, the solution mentioned above may not always be effective in controlling these modes in the stop band. The solutions available in the literature are either shown to be effective for PCs or for a specific type of BCs and specific position of the resonant elements in LRMs. Therefore, a more systematic investigation for LRMs is needed to assess (i) how the stop band is preserved in a general finite LRM system with different BCs and (ii) how the modes in the stop band can be controlled. This would help to design efficient LRM solutions that comply with possible space, manufacturing, cost, aesthetic constraints, and the BCs applied in any industrial application.

The objective of the presented study is twofold: firstly, to investigate the influence of different BCs on the stop band effect for a finite LRM beam. A particular focus is given to the influence of modes in the stop band. Secondly, to explore the effectiveness and limitations of the solutions available in the literature to control modes in the stop band. 
In the first part of this paper, a systematic numerical parameter analysis is provided on several LRM beam configurations and for different BCs. A Timoshenko beam with tuned vibration absorbers (TVAs) serves as a computational not demanding model to describe a generic LRM beam configuration. Such a simple model helps to gain insight into the underlying mechanism, which might be hindered by the complexity involved when using more elaborated LRM systems. Finite element (FE) models are applied for both the UC $[29,30,31,32]$ as well as the LRM beam. UC analysis and modal analysis are used to predict the stop band behaviour in the infinite and finite beam, respectively. Next, three methods are explored to preserve the predicted stop band in the finite LRM beam: (i) tuning the resonance frequency, (ii) the number and (iii) the position of the resonant elements in the finite beam. The first method investigates how the perturbation of the properties of the resonant elements affects the modes in the stop band. This is inspired on the method from the literature, in which perturbation of the material properties and size of the scatterers was used to avoid modes in the stop band. The second and third method are based on the aforementioned studies in the literature and are now extended to different types of BCs and target frequency ranges, such that the effectiveness and limitations of the methods are further investigated.

In the second part, the numerical findings are experimentally validated on an actual LRM beam, which consists of an aluminium beam as host structure and beam-like resonators made from polymethyl methacrylate (PMMA) as resonant structures [16]. A frequency response function (FRF) based comparison is used to correlate the experimental results to the numerical results.

This paper is organised as follows: after the introduction, section 2 describes the LRM beam used in the parameter study, the performance indicators to evaluate the different configurations, and the parameters analysed in the investigation. Section 3 discusses the results from the parameter investigation. In section 4 , three methods are analysed to control edge modes in the predicted stop band frequency range. In section 5 , a numerical and experimental validation of the parameter investigation is carried out for a real LRM beam. The paper ends with the conclusions.

\section{Problem definition}

This section describes the host structure and the resonant elements used to compose the LRM beam in the investigations as well as the metrics and the parameters used.

\subsection{LRM beam}

Table 1 summarises the material properties and the dimensions of the UC of the LRM beam. The UC of an infinite periodic LRM beam is modelled with the FE method. A Timoshenko beam model is chosen to include the effects of shear deformation and rotatory inertia in a UC with a slenderness ratio equal to 10 [33]. The resonant element is modelled as a TVA. The mesh of the FE model consists of 20 elements 
Table 1: Material and geometric characteristics of the UC.

\begin{tabular}{ccc}
\hline Name & Symbol & Value \\
\hline Young's Modulus & $\mathrm{E}$ & $210 \mathrm{GPa}$ \\
Density & $\rho$ & $7800 \mathrm{~kg} / \mathrm{m}^{3}$ \\
Poisson's ratio & $\nu$ & 0.3 \\
Height & $\mathrm{H}$ & $10 \mathrm{~mm}$ \\
Width & $\mathrm{W}$ & $10 \mathrm{~mm}$ \\
Shear coefficient & $k_{s}$ & $5 / 6$ \\
UC length & $L_{U C}$ & $0.1 \mathrm{~m}$ \\
\hline
\end{tabular}

per UC. This mesh ensures numerical convergence of the results in the frequency range of interest, with a relative error smaller than $1 \mathrm{e}-6$. The UC length, together with the cross-section, the material properties and the mesh are kept constant throughout the whole investigation. The finite beam is obtained by adding sequentially a finite number of UCs along the axis of the beam and applying a set of BCs at its extremities.

\subsection{Stop band evaluation in infinite and finite LRM beams}

An undamped FE model of the UC with periodic Bloch-Floquet boundary conditions [4, 30] is used to predict stop band behaviour in an infinite LRM beam. Stop band behaviour in the finite LRM beam is studied by calculating the natural frequency distribution through a modal analysis and by calculating the forced response of the LRM beam under a point force excitation and with different BCs. The stop band frequency limits are found by tracking the phase of the relative motion between the resonant elements and the host structure: the resonant element moves in-phase with respect to the host structure until the lower limit of the stop band and in anti-phase after the stop band. As a result, a phase shift of $\pi$ rad occurs between the lower and the upper stop band bound [34].

\subsection{Considered configurations for the numerical parameter investigation}

A parameter study is performed to assess the influence of the BCs on the stop band behaviour in the finite LRM beam as compared to the stop band predicted in the infinite LRM system. The following BCs are investigated:

- Simply supported (SS): fixed translations and free rotations;

- Clamped (CC): fixed translations and fixed rotations;

- Free $(\mathrm{FF})$ : free translations and free rotations; 
- Restraint (RR): grounded translational and rotational springs with adjustable stiffness.

The SS BC is chosen since the natural frequencies of a finite beam with SS BC are known to correspond to fixed locations on the dispersion curves of the corresponding infinite periodic structure [35, 36]. As a result, no natural frequencies of the structure occur in the predicted stop band. CC and FF BCs are chosen because they are limit cases. The RR BCs allow modelling the most generic BC case between the extremes, $\mathrm{CC}$ and FF, by varying the stiffness of the springs.

The length of the finite beam, and the added mass and tuned frequency of the TVAs are varied to evaluate how these parameters affect the natural frequency distribution and the vibration attenuation in the predicted stop band. The length of the finite beam is expressed in terms of the number of UCs. The added mass is given in percentage with respect to the mass of the beam. The tuned frequency of the TVA is normalised in terms of frequency ratio [34], defined by eq. (1):

$$
f_{\text {ratio }}=\frac{f_{\text {res }}}{f_{\lambda / 2}}
$$

where $f_{\text {ratio }}$ expresses the ratio between the tuned frequency of the resonator $f_{\text {res }}$ and the frequency $f_{\lambda / 2}$ at which half of the structural wavelength of the bare beam equals the distance between two consecutive resonant elements. For bending waves in a Timoshenko beam, this frequency is found by setting the structural wavelength $\lambda$ in the wavenumber definition $k=2 \pi / \lambda$ equal to $2 L$ and solving eq. (2) [37] for the frequency $f_{\lambda / 2}$ :

$$
f_{\lambda / 2}^{4}-f_{\lambda / 2}^{2} \frac{1}{(2 \pi)^{2}}\left(\frac{k_{s} G A}{I \rho}+\frac{k_{s} G+E}{\rho} \frac{\pi^{2}}{L^{2}}\right)+\frac{k_{s} E G}{\rho^{2}} \frac{1}{16 L^{4}}=0 .
$$

All the analysis are performed in the subwavelength frequency region up to $f / f_{\lambda / 2}=1$.

\section{Results of the parameter investigation}

In this section, the results of the parameter investigation are presented and discussed. The influence of the BCs on the predicted stop band is analysed in two steps. First, the natural frequency distribution of a finite LRM beam with different BCs is compared to the predicted stop band frequency range. LRM beams of different (i) length and with different (ii) added masses and tuned frequencies are considered. Secondly, the forced response of a chosen finite LRM beam configuration is analysed.

\subsection{Influence of the BCs on the natural frequency distribution in LRM beam of varying length}

The length of the beam is varied by changing the amount of UCs. The added mass per UC and the tuned frequency are kept constant. The configurations analysed consist of LRM beams with TVAs adding $20 \%$ of mass and tuned to frequency of $f_{\text {ratio }}=0.5$. The length of the beam is varied from 1 UC to 20 UCs. $\mathrm{SS}, \mathrm{FF}$ and CC BCs are applied to the LRM beams. The natural frequency distribution of these LRM beam configurations, as a function of the number of UCs they are composed of, is depicted in Figure 1. The stop 


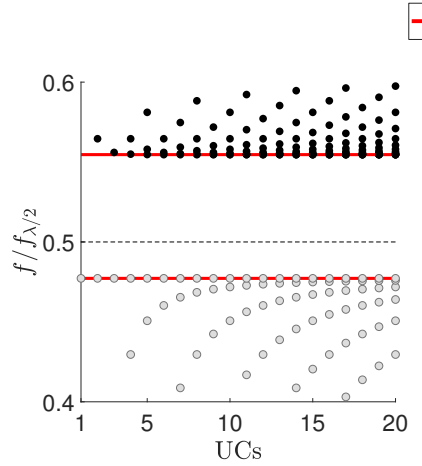

(a)

\section{-Predicted stop band---Tuned frequency $\circ$ In-phase $\bullet$ Anti-phase}
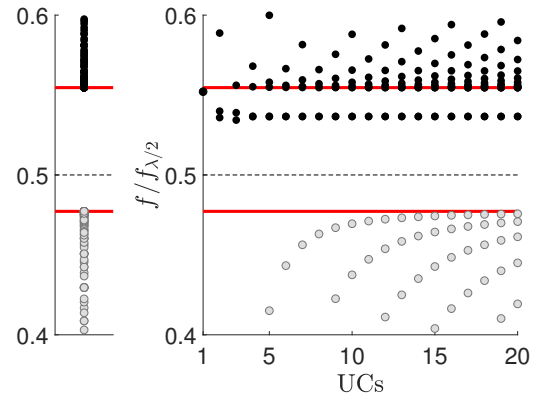

(b)
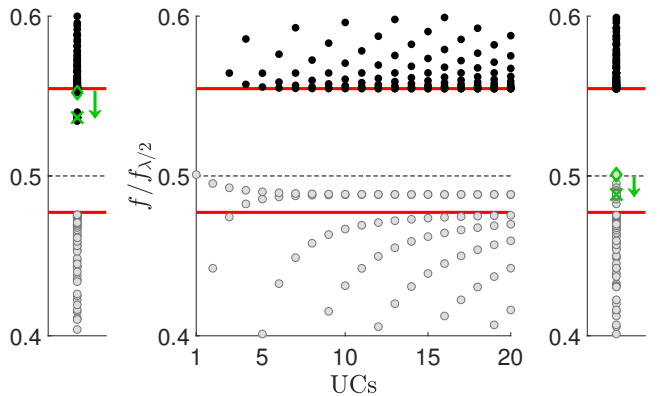

(c)

Figure 1: Natural frequency distribution for finite LRM beam of different length. Configurations: (a) SS, (b) FF and (c) CC BCs. The natural frequencies of all 20 lengths of the beam considered are plotted together in the graph at the right hand side of (a), (b) and (c). The green $(\rightarrow)$ in these graphs indicate the shift in frequency of the natural frequencies with different number of UCs considered in the LRM beam. The green $\diamond$ and $\times$ indicate the natural frequencies of the LRM beam consisting of 1 and $20 \mathrm{UCs}$, respectively.

band for the infinite periodic structure occurs between $f / f_{\lambda / 2}=0.47$ and $f / f_{\lambda / 2}=0.545$. As can be seen in Figure 1(a), no eigenmodes for the beam with SS BCs occur in the stop band frequency region predicted by the UC analysis, independently of the length of the beam. The TVAs move with respect to the beam in-phase (light grey circles) below the lower limit of the predicted stop band region, and in anti-phase (black circles) after the stop band region, agreeing with the findings of [34]. The modal density around the lower and upper limit of the stop band increases with the number of UCs included in the LRM beam, agreeing with the findings in $[35,38,36,39]$. The results for FF and CC BCs, in Figure 1(b) and 1(c), differ from the results for the SS BCs configuration. Natural frequencies are present in the predicted stop band frequency range with a behaviour that differs according to the BCs applied. The modes in the stop band in the case of FF BCs have TVAs moving in anti-phase motion with respect to the beam. For CC BCs, an in-phase motion between TVA and beam is present. The modes in the stop band region are called edge modes and come in pairs. They develop from the edges of the beam, where they have the highest amplitude, and they decay rapidly with the distance from the edge, as discussed in [23] and as depicted in Figure 2.

For a beam composed of a limited number of UCs, the frequencies of the edge modes change within the predicted stop band as a function of the number of UCs considered. When the beam is composed of more than $19 \mathrm{UCs}$, the addition of more UCs to the beam does not affect anymore the frequency at which these edge modes occur. Therefore, the length of the LRM beam is kept fixed at 20 UCs in the following analyses. As such, the frequencies of the edge modes are no longer dependent on the length of the beam. The objective is then to highlight how other parameters, such as added mass and tuned frequency, affect the edge modes in LRM beams with different BCs. Considering an LRM beam with 20 UCs, the edge modes 


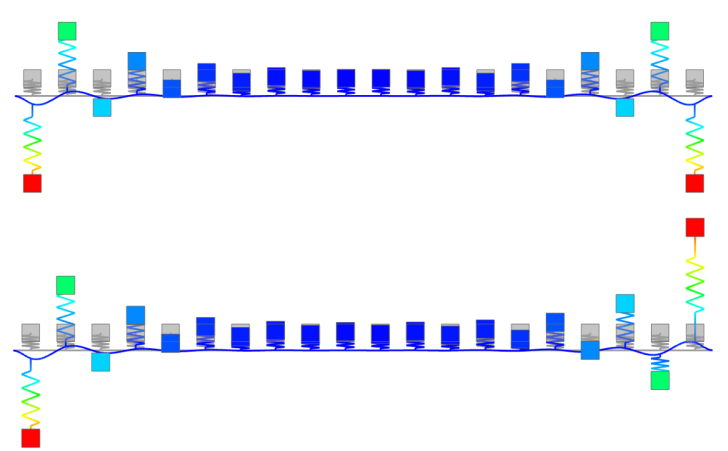

(a)

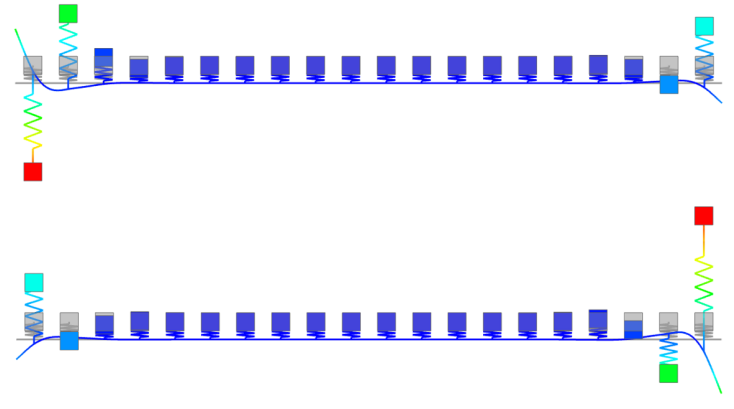

(b)

Figure 2: (a) Clamped edge modes at $f / f_{\lambda / 2}=0.48$ for CC BCs and (b) free edge modes at $f / f_{\lambda / 2}=0.528$ for FF BCs. The undeformed LRM beam is represented by the light grey colour.

3.2. Influence of the BCs on the natural frequency distribution in LRM beam of varying mass and tuned frequency of the TVAs

LRM beams with mass ratios of $20 \%$ and $80 \%$ are considered, and the tuned frequency of the TVAs is varied in the different analyses. The results from the UC analysis, depicted in Figure 3, are in agreement with [34]: (i) the width of the stop band increases with the $f_{\text {ratio }}$ and (ii) for the same $f_{\text {ratio }}$, the system with higher added mass has a wider stop band. Varying the $f_{\text {ratio }}$, the natural frequencies for SS BCs are in the pass band predicted by the UC analysis, as can be seen in Figure 3(a)-(e). Based on the results shown in Figure 3(b)-(f) and 3(c)-(g) it is observed that:

- for FF BCs, the free edge modes have an anti-phase motion for $f / f_{\lambda / 2}>f_{\text {ratio }}$, and an in-phase motion otherwise;

- for $\mathrm{CC}$ BCs, the clamped edge modes have an in-phase motion for the whole $f_{\text {ratio }}$ range analysed;

- the frequencies at which the edge modes occur for both configurations are sensitive to the $f_{\text {ratio }}$ and the added mass. The higher the $f_{\text {ratio }}$ and the added mass, the larger the distance of the edge modes from the stop band limits.

Applying the RR BCs allows to extend the analysis to combinations of BCs in between the FF and the CC. The spring stiffness is varied in a range from $1 \mathrm{e}-1 \mathrm{~N} / \mathrm{m}(\mathrm{Nm} / \mathrm{rad})$ to $1 \mathrm{e} 15 \mathrm{~N} / \mathrm{m}(\mathrm{Nm} / \mathrm{rad})$ for the translational (rotational) springs. In Figure 3(d)-(h) multiple RR BCs configurations solutions per $f_{\text {ratio }}$ are plotted. Edge modes occur in the predicted stop band for every configuration of BCs except for the SS BCs. The reason for this is that the bending modes of a SS BCs beam can be described only by the propagating solutions of the beam equation [40]. The solution of the UC analysis of an undamped UC model 


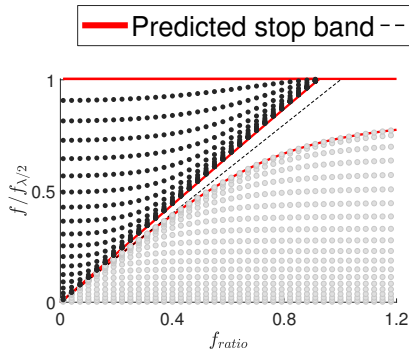

(a)

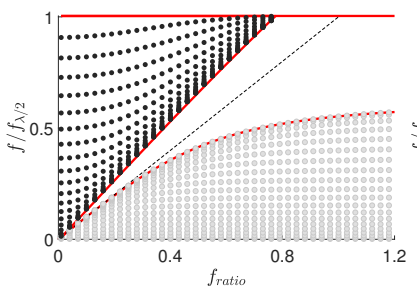

(e)

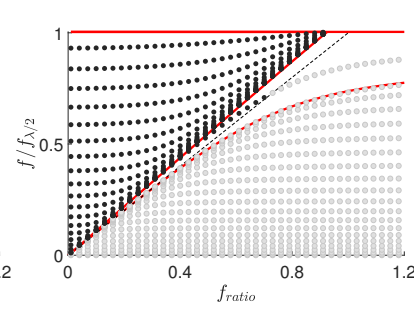

(b)

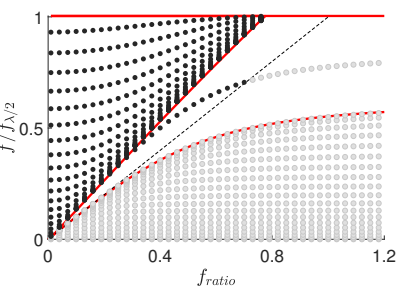

(f)

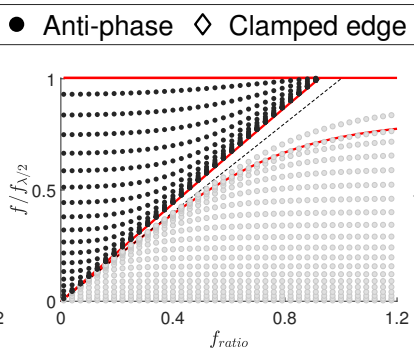

(c)

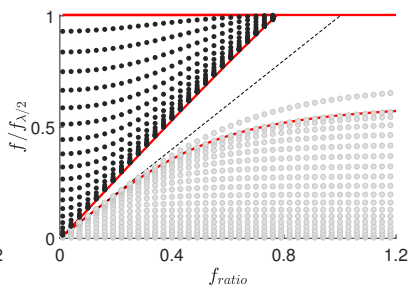

(g)

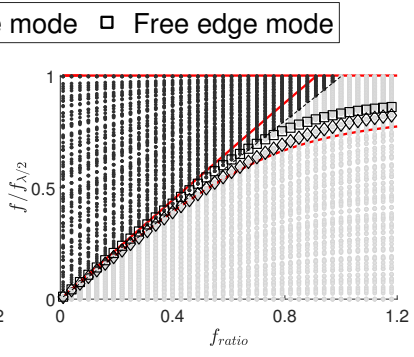

(d)

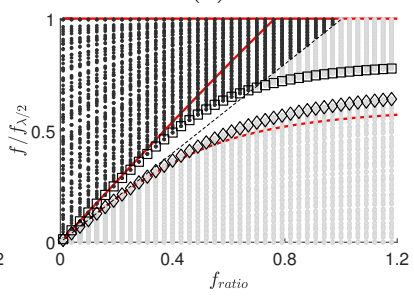

(h)

Figure 3: Natural frequency distribution for finite LRM beam with different tuned frequencies of the TVAs. Configurations: (a)-(e) SS, (b)-(f) FF, (c)-(g) CC and (d)-(h) RR BCs. Top row $20 \%$ of added mass and bottom row $80 \%$ of added mass. The black square $(\square)$ and diamond $(\diamond)$ in $(\mathrm{d})-(\mathrm{h})$ indicate the free edge and the clamped edge mode respectively for CF BCs.

indicates in which regions no freely propagating waves are found and modes of SS beam will not be present in the stop band [36]. For all other combinations of BCs, the decaying waves together with the propagative waves are solutions of the beam equation and are required to represent the edge modes. The set of waves that represent the edge modes is not described by the UC modelling. As a result, edge modes can occur in the predicted stop band. Other authors describe this effect as lattice symmetry-breaking, which occurs when a discontinuity in the lattice due to defects [25] or FF BCs in PCs [26] breaks the periodicity in the infinite periodic structure.

When applying clamped-free $(\mathrm{CF}) \mathrm{BCs}$, both clamped and free edge modes are present in the stop band. The solution for CF BCs is highlighted in Figure 3(d)-(h) by the two curves with black square ( $\square$ ) and diamond $(\diamond)$ symbols, which indicate the free and clamped edge modes, respectively. As can be seen from the results, this case representes the limit case and these curves limit the frequency zone where no modes are present in the stop band. For $f_{\text {ratio }}<0.1$, independently of the BCs and the added mass, the natural frequency distribution is in good agreement with the UC analysis results, as also observed by [41, 42].

\subsection{Structural response of a finite LRM beam}

To provide an insight into how the edge modes influence the structural attenuation in the stop band frequency range, the structural response of the LRM beam to point-force excitation is investigated and this is repeated for different excitation locations. An LRM beam with TVAs tuned at $f_{\text {ratio }}=0.5$ and with an added mass of $20 \%$ is chosen. SS and CF BCs are applied. The latter BCs are chosen because both free 
and clamped edge modes occur in the stop band. The clamped boundary is on the left side of the beam, which coincides with the origin of the chosen reference system (Figure 4). Structural damping of 1\% in the beam and critical damping of $0 \%, 0.1 \%$, and $1 \%$ in the TVAs is considered. The point force load is moved

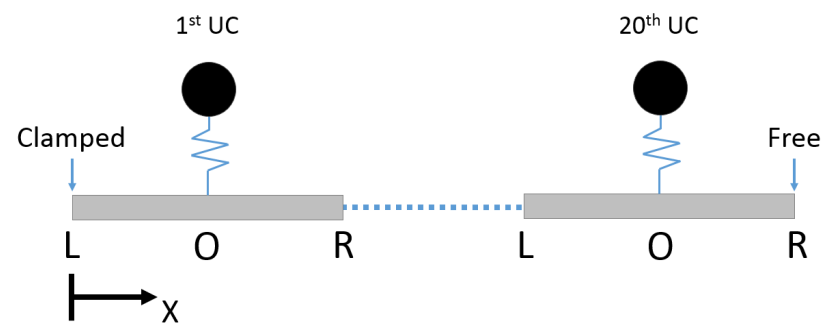

Figure 4: Sketch of the LRM beam with CF BCs.

along the beam length. In each simulation, an excitation of magnitude $1 \mathrm{~N}$ is applied to a single node of the mesh. This is repeated for every node on the mesh, except for the ones at the boundaries. For each simulation, the average kinetic energy $(\overline{K E})$ of the beam is evaluated using eq. (3):

$$
\overline{K E}=\frac{1}{2} m_{b}<v(\omega)^{2}>,
$$

where $m_{b}$ is the mass of the beam without TVAs and $\left\langle v(\omega)^{2}\right\rangle$ indicates the square of the root means square (RMS) value of the out-of-plane velocity, which is evaluated for each frequency of interest for all the response nodes in the beam, excluding the nodes related to the point masses of the TVAs.

Figure 5 visualises the evolution of the $\overline{K E}$ of the LRM beam for the combination of excitation frequency and force location. For SS BCs, the frequency region of vibration attenuation is independent of the location of the excitation. In the case of CF BCs, the level of vibration increases in the stop band. When the force is within a certain distance from the boundaries, the edge modes are excited. If the beam is sufficiently long as is the case here, the force acting close to one boundary can excite only the edge mode corresponding to that boundary. At a distance sufficiently far away from the boundaries, the structural response of the beam with SS and CF BCs is similar in the stop band region. To better visualise this behaviour at the frequencies of the clamped and free edge modes, Figure 6 repeats the $\overline{K E}$ in function of the position of the point force on the beam at these specific frequencies. It can be seen in Figure 6(a) that, for the case of undamped TVAs, the $\overline{K E}$ of the CF beam is higher than the $\overline{K E}$ of the SS beam for an excitation within the first 8 UCs from the clamped boundary. This indicates that the in-phase clamped edge mode is excited for an excitation within these UCs. Similarly, inspection of Figure 6(b) shows that the anti-phase free edge mode is excited when the force is applied within 4 UCs from the free edge, in the case of undamped TVAs. With higher damping applied in the TVAs, the distance from the boundaries, within which the edge modes can be excited, is reduced (Figure 6). 
- Predicted stop band

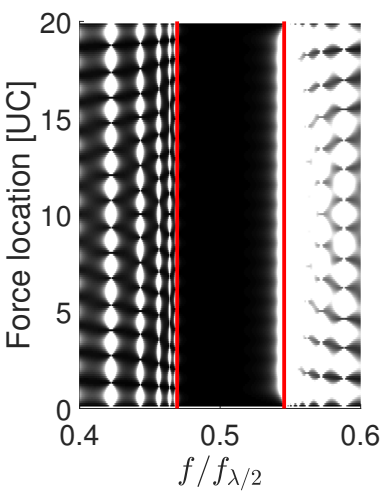

(a)

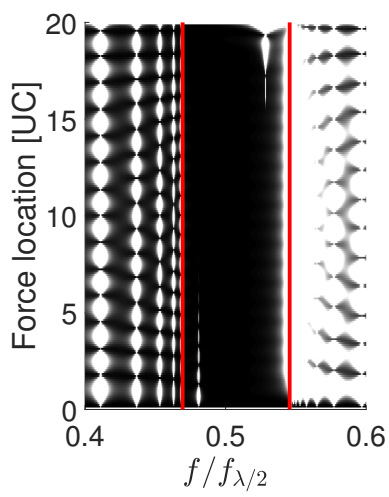

(b)

Figure 5: Average kinetic energy of the LRM beam due to a point force excitation acting at different positions on the beam and for different excitation frequencies. The forced response is plotted for the case of $1 \%$ of structural damping in the beam and undamped TVAs. Configurations: (a) SS and (b) CF BCs. The origin of the y-axis for (b) corresponds to the clamped boundary according to Figure 4 .

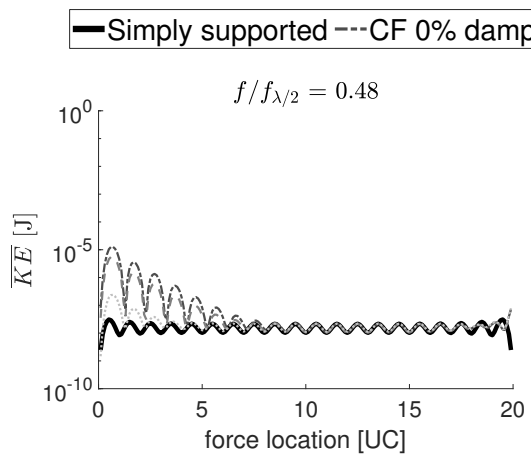

(a)

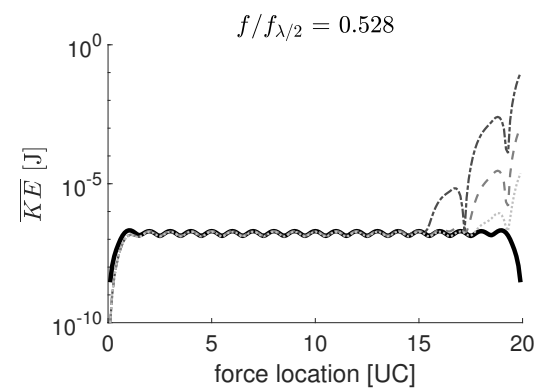

(b)

Figure 6: Average kinetic energy of the LRM beam due to a point force excitation acting at different positions on the beam at the frequencies of the (a) clamped and (b) free edge mode. The forced response is plotted for different amount of critical damping in the TVAs, while keeping $1 \%$ of structural damping in the beam. 


\section{Reduction of the effect of the edge modes in the stop band}

In previous sections it was shown that edge modes depend on the BCs, beam length, and the tuned frequency and added mass of the TVAs. This section explores the effectiveness and the limitations of the solution available in literature for reducing the effect of the edge modes in the stop band for a general LRM beam.

A local variation of the mechanical properties in PCs shows to influence the frequency of the modes occurring in the predicted stop band [26, 28]. Following a similar approach, the local properties of the resonant additions in a LRM beam are varied by changing three different parameters: (i) the tuned frequencies, (ii) the number and (iii) the position of the TVAs in the beam. Varying one of these parameters can be beneficial when one of the following industrial constraints are present:

- varying the tuned frequency of the TVAs, where constraints on the positions of the resonant elements are present or where the periodicity on the host structure needs to be preserved;

- varying the number of TVAs, where space constraints in the host structure are present;

- varying the position of the TVAs, where design constraints on the resonant elements are applied.

A CF BCs beam with TVAs adding $20 \%$ of mass is used in the investigation. The analyses are performed for different values of $f_{\text {ratio }}$, focusing on values of $f_{\text {ratio }}>0.1$, given that for lower values, a good agreement between the stop band prediction and the natural frequency distribution is found.

\subsection{Variation of the tuned frequency of the TVAs}

The tuned frequencies of all TVAs in the beam are allowed to be varied independently, to remove the edge modes in the predicted stop band. A derivative based optimizer [43] is used and the goal function is given by eq. (4):

$$
\left\{\begin{array}{l}
\left\{\left(\Delta f_{U B}\right)^{2}+\left(\Delta f_{L B}\right)^{2}\right\}_{\text {min }}, \\
\Delta f_{x}=f_{S B_{x}}-f_{E M_{x}}, x=\{U B, L B\} ;
\end{array}\right.
$$

where $S B$ and $E M$ stand for stop band and edge mode, respectively, while $f_{S B_{x}}$ and $f_{E M_{x}}$ indicate the frequency of the lower bound $(x=L B)$ and of the upper bound $(x=U B)$ of the stop band, and the closest edge mode to the corresponding stop band bound, respectively. A solution is sought for different $f_{\text {ratio }}$ in the interval 0.1 to 0.99 with a step of 0.1 .

Figure 7(a) depicts the optimal tuned frequency distribution of each TVA in the beam as a function of the $f_{\text {ratio. }}$. The colour scale indicates the relative change with respect to the original tuned frequency. A negative value indicates a lower tuned frequency with respect to the original, whereas a positive value indicates a higher tuned frequency with respect to the original. For $f_{\text {ratio }}<0.7$, the optimal value for TVAs 


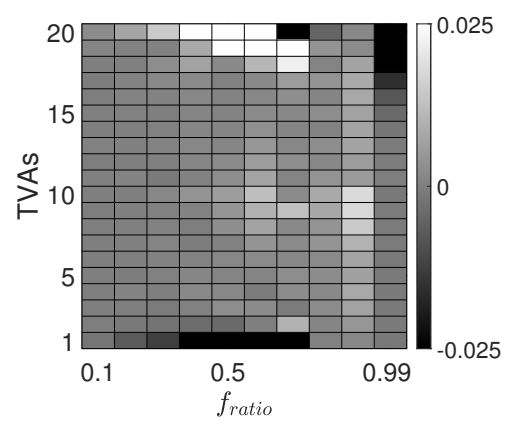

(a)

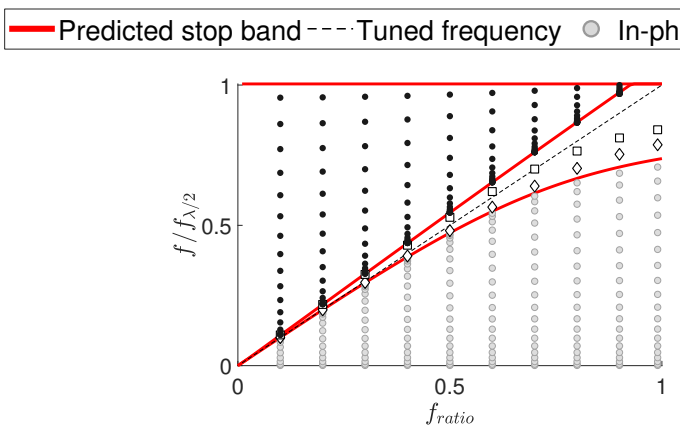

(b)
Anti-phase $\diamond$ Clamped edge mode $\square$ Free edge mode

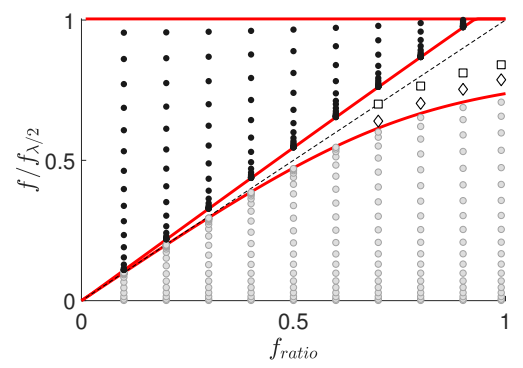

(c)

Figure 7: (a) Optimal tuned frequency distribution of the TVAs for different values of $f_{\text {ratio. }}$. The colour scale indicates the relative change with respect to the original tuned frequency. Natural frequency distribution for different values of $f_{\text {ratio }}$ for the (b) original and (c) optimised configurations. 
close to the clamped edge changes to a lower tuned frequency. For TVAs close to the free edge, the tuned frequency changes to higher values. Mainly the tuned frequency of the first one or two TVAs closest to the edge changes. The effect of the optimisation on the effective stop band width is shown in Figure 7(b) and (c): the natural frequency distribution is shown for (i) the original configuration, with all TVAs tuned to the same target frequency, and (ii) the optimised configuration, for each $f_{\text {ratio }}$, with each TVA tuned to the target frequencies found in Figure 7(a). The edge modes, which were present in the stop band in the original configuration for $f_{\text {ratio }} \leq 0.7$, are no longer present in the optimised configurations. For $0.7<f_{\text {ratio }}<1$, the locally resonant stop band starts coupling with the Bragg stop band and behaving as a hybrid Bragg stop band [42]. Therefore, the stop band becomes more sensitive to the perturbation of the lattice periodicity and tuning the frequency of the TVAs does not suffice anymore to prevent edge modes from appearing in the predicted stop band for the optimised configurations.

\subsection{Variation of the number of TVAs on the beam}

As a next parameter, the number of TVAs on the LRM beam is considered. For this study, the TVAs on the beam are eliminated in pairs at each boundary to keep a symmetric mass distribution on the beam. A maximum of 10 out of 20 TVAs, 5 on each side of the beam, are removed. If more TVAs are removed, a clear stop band behaviour in this finite LRM beam is no longer observed.

The results are depicted in Figure 8 for $f_{\text {ratio }} 0.2,0.5,0.8$ and 0.9 . The number of TVA that need to be removed varies as a function of $f_{\text {ratio }}$ : for $f_{\text {ratio }}=0.2$, either 1 or 3 TVAs can be removed from each side of the beam; for $f_{\text {ratio }}=0.5,2$ TVAs need to be removed on each side; and for $f_{\text {ratio }}=0.8,4$ TVAs need to be removed on each side. For $f_{\text {ratio }}=0.9$, edge modes are still present in the predicted stop band after removing up to 5 TVAs from each side of the beam.

\subsection{Variation of the position of the TVAs on the beam}

As a last method, the position of TVAs on the beam is varied. All the TVAs are moved along the axis of the beam within their UC. The same distance among the TVAs is maintained and, thus, the predicted stop band for the infinite structure is not affected. In finite LRM beams, however, the positions of the TVAs may strongly affect the dynamics of the entire LRM beam [27]. The position of the TVAs is varied within a UC from the left side (L) to the right side (R), as sketched in Figure 4. Position $(\mathrm{O})$ indicates the original position of the TVAs, being in the middle of the UC. During the analysis, the TVA is positioned on every node of the UC. The results are presented for for $f_{\text {ratio }} 0.2,0.5,0.8$ and 0.9 in Figure 9 . The frequencies of the edge modes show to be sensitive to the position of TVAs in the UC. In particular, the higher the $f_{\text {ratio }}$, the more TVAs need to be moved from L to R, according to Figure 4 . This corresponds to the TVAs moving away from the clamped edge and closer to the free edge. The edge modes are no longer present in the stop band for $f_{\text {ratio }}=0.2$ and $f_{\text {ratio }}=0.5$. 


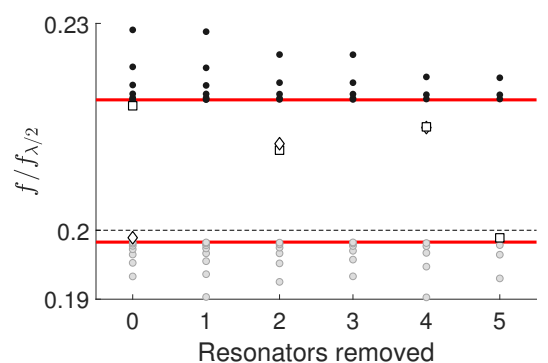

(a)

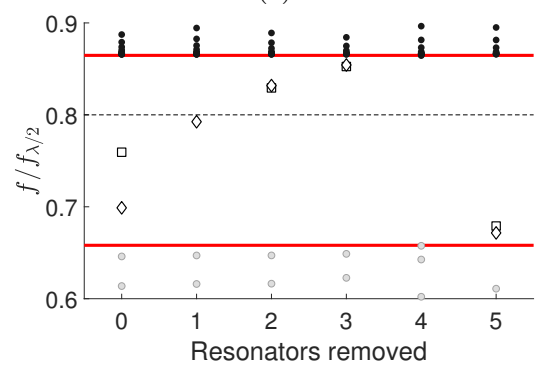

(c)

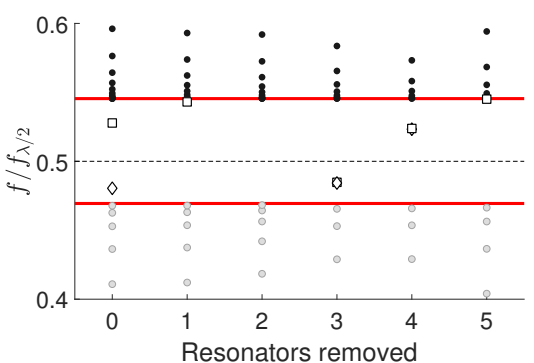

(b)

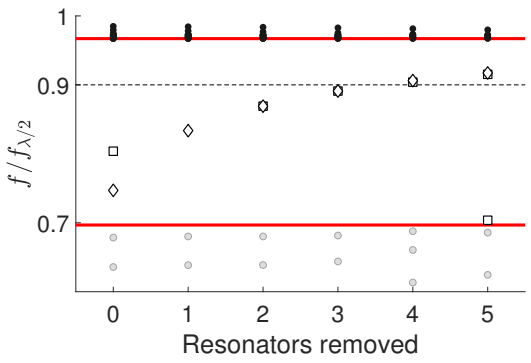

(d)

Figure 8: Natural frequency distribution for the CF LRM beam with TVAs removed in pair from each boundaries of the beam. Configurations: (a) $f_{\text {ratio }}=0.2$, (b) $f_{\text {ratio }}=0.5$, (c) $f_{\text {ratio }}=0.8$ and (d) $f_{\text {ratio }}=0.9$.

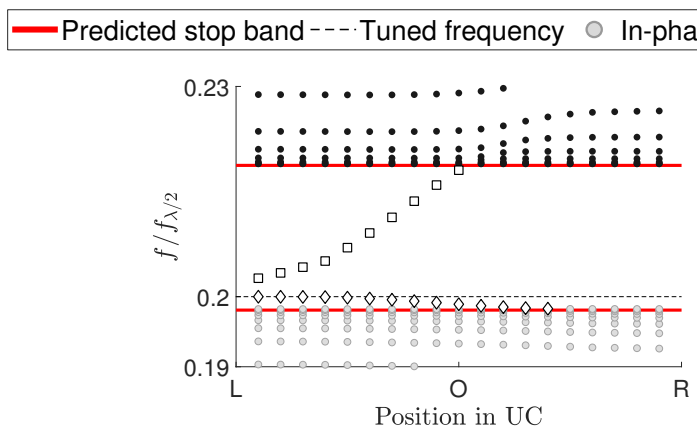

(a)

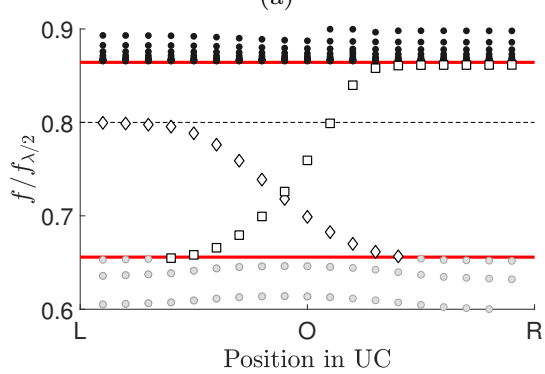

(c)
Anti-phase $\diamond$ Clamped edge mode $\square$ Free edge mode

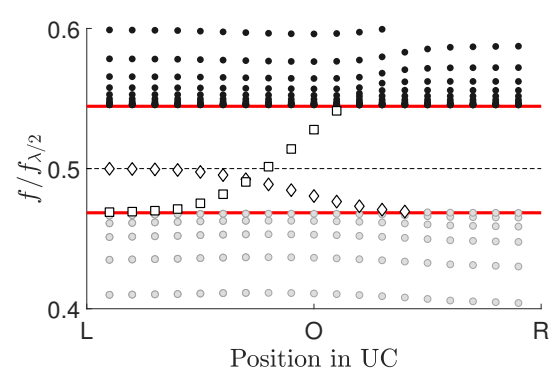

(b)

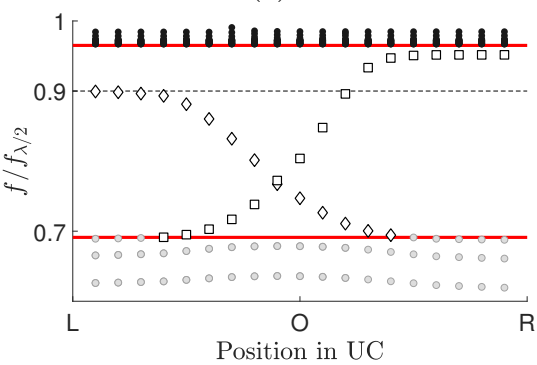

(d)

Figure 9: Natural frequency distribution for the CF LRM beam with all TVAs moved in the UC. Configurations: (a) $f_{\text {ratio }}=$ 0.2 , (b) $f_{\text {ratio }}=0.5$, (c) $f_{\text {ratio }}=0.8$ and (d) $f_{\text {ratio }}=0.9$. 
Similarly as in the case of sections 4.1 and 4.2 for $f_{\text {ratio }}$ close to 1 , the edge modes are difficult to avoid within the predicted stop band. For $f_{\text {ratio }}=0.8$ and $f_{\text {ratio }}=0.9$, only the free edge mode is still present in the stop band, although close to the upper limit of the stop band. Comparing the results of Figure 7 and 8 with the results in Figure 9 for $f_{\text {ratio }}>0.8$, varying the position of the TVAs shows to be a more effective solution to control the edge modes.

\section{Validation of the numerical findings}

To validate the numerical findings of the previous sections, one specific LRM beam configuration is chosen. The design of the host structure, resonant structures, LRM configuration and experimental setup are presented in the following sections. Numerical and experimental results are then discussed.

\subsection{Definition of the LRM beam configuration}

The dimensions of the aluminium host structure are reported in Table 2 [44]. The combination of material, geometry of the cross-section and length of the host structure is chosen to have a flexible system with pronounced modal behaviour at the low-frequency range below $1 \mathrm{kHz}$.

The material parameters of the host structure are obtained from a plate of dimensions $276 \times 563 \times 2 \mathrm{~mm}$, cut out from the same panel of which the host structure is constructed. The density is retrieved through a weight measurement, while Young's modulus, Poisson's ratio and structural damping are obtained based on the correlation between the mode shapes retrieved with an FE model and the experimentally measured mode shapes. The FE model is built in NX Nastran 12 and composed of 9729 linear CQUAD4 shell elements, [45] and free BCs are applied. The sample is tested for freely suspended BCs and a roving hammer approach and one lightweight accelerometer, type PCB 352A24 with weight $0.8 \mathrm{~g}$, are used. The retrieved material properties are reported in Table 2 . The difference between the numerically calculated and experimentally retrieved mode shapes is less than $1 \%$.

Table 2: Updated material properties and geometric characteristics of the host structure.

\begin{tabular}{ccc}
\hline Name & Symbol & Value \\
\hline Young's Modulus & $\mathrm{E}$ & $68.07 \mathrm{GPa}$ \\
Density & $\rho$ & $2690.2 \mathrm{~kg} / \mathrm{m}^{3}$ \\
Poisson's ratio & $\nu$ & 0.325 \\
Structural damping & $\eta$ & $0.25 \%$ \\
Length & $\mathrm{L}$ & $1080 \mathrm{~mm}$ \\
Height & $\mathrm{H}$ & $2 \mathrm{~mm}$ \\
Width & $\mathrm{W}$ & $30 \mathrm{~mm}$ \\
\hline
\end{tabular}


The resonant structure is similar to the one proposed in [16], which consists of a cantilever-like beam and an end-point mass. The geometry is depicted in Figure 10(a). The resonant elements are made by

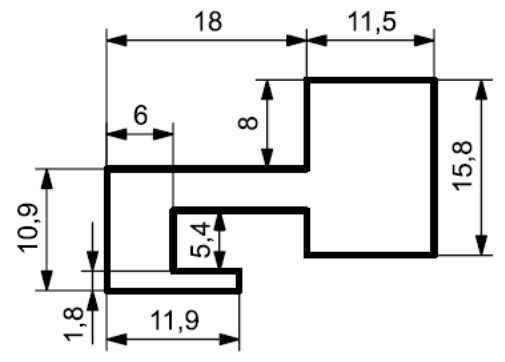

(a)

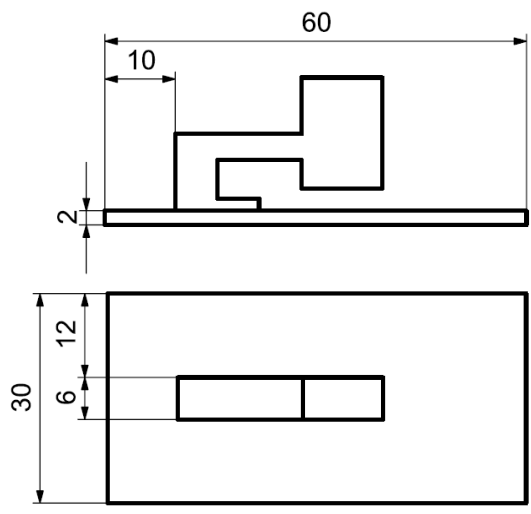

(b)

Figure 10: Geometry of the resonant elements and of the UC assembly made by host structure and resonant element. (a) Side view of the resonant element. (b) Side and top view of the UC assembly. The dimensions are given in mm.

laser cutting from a PMMA panel. The material properties of the PMMA are reported in Table 3. The method to retrieve the material parameters can be found in [16]. The resonant element is designed to have an out-of-plane mode at $800 \mathrm{~Hz}$ to target the bending modes of the beam below $1 \mathrm{kHz}$. An FE model of the resonant element is used to predict the resonance frequency. The numerical model consists of 472 linear CHEXA8 solid elements [45] and clamped BCs applied at the base of the resonant element.

The resonance frequency of the resonant element is retrieved by attaching it to a shaker and measuring the velocity at the tip of their end-point mass using a Polytec PSV-500 Scanning Laser Doppler Vibrometer (SLDV) [16]. A set of 35 resonators is measured, of which a subset of 18 resonant elements is chosen. These resonant elements are chosen to have the smallest spread on their resonance frequencies, resulting in a resonance frequency of $800 \mathrm{~Hz}$ with a spread of $\pm 0.9 \%$.

Table 3: Updated material properties of the PMMA resonant elements.

\begin{tabular}{ccc}
\hline Name & Symbol & Value \\
\hline Young's Modulus & $\mathrm{E}$ & $4.85 \mathrm{GPa}$ \\
Density & $\rho$ & $1188.4 \mathrm{~kg} / \mathrm{m}^{3}$ \\
Poisson's ratio & $\nu$ & 0.31 \\
Structural damping & $\eta$ & $5 \%$ \\
\hline
\end{tabular}



$f / f_{\lambda / 2}=0.542$ and $f / f_{\lambda / 2}=0.644$.

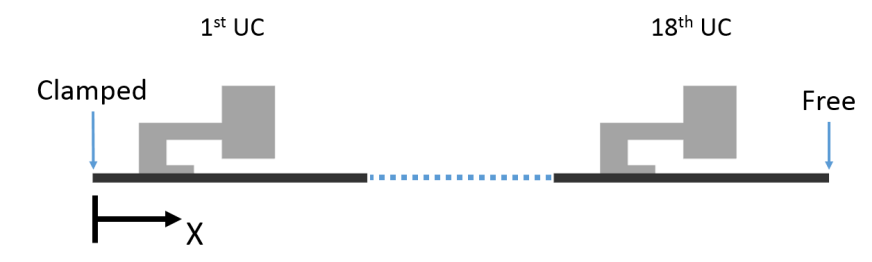

Figure 11: Sketch of the LRM beam with CF BC.

The resonant element is added onto a UC of dimensions $60 \times 30 \mathrm{~mm}$, as depicted in Figure 10(b), to be subwavelength at $800 \mathrm{~Hz}$ and to guarantee stop band behaviour. With these UC dimensions, the tuned frequency of the resonant structure corresponds to a $f_{\text {ratio }}=0.633$. The resonant elements are attached to the host structure with superglue. Each resonant element adds about $22 \%$ of mass to the UC. The FE model of the LRM UC consists of 210 linear CQUAD4 shell elements for the host structure and 472 linear CHEXA8 solid elements for the resonant element. A stop band for the bending waves opens up between

The finite LRM beam consists of 18 UCs repeated along its longitudinal axis. The BCs applied on the beam are SS and CF. The LRM beam is oriented according to Figure 11. The left-hand side of the beam is clamped, while the right end side is left free.

Figure 12(a) depicts the natural frequency distribution of the simulated finite LRM beam with SS and CF BCs. When SS BCs are applied, no edge modes are present in the predicted stop band, as observed in section 3. The LRM design targets a stop band specifically for bending waves, hence, torsional modes still occur in the predicted stop band. When CF BCs are applied, besides the torsional modes, two edge modes are present in the stop band. These edge modes occur at $f_{\text {ratio }}=0.5743$ for the clamped edge mode and $f_{\text {ratio }}=0.6285$ for the free edge mode. The mode shapes of the edge modes are represented in Figure 12(b). An in-phase mode corresponds to the clamped edge mode, and an anti-phase mode corresponds to a free edge mode.

\subsection{Experimental setup}

For the experimental validation, the LRM beam is clamped with a heavy clamp of about $15 \mathrm{~kg}$ to a rigid aluminium wall (Figure 13). Impact tests with hammer excitation are used to excite the LRM beam.

The vibration response of the beam is measured with an SLDV. The response of the beam is evaluated in 57 points distributed over 19 sections of 3 points each: 1 at the centre and 2 at the side of the section. These points are located at the edge of each UC. The first section is located close to the clamped boundary, whereas the last section is located at the free boundary. Two excitation locations are chosen: the centre point of the first section and the centre point of the last section. These locations allow exciting both edge 


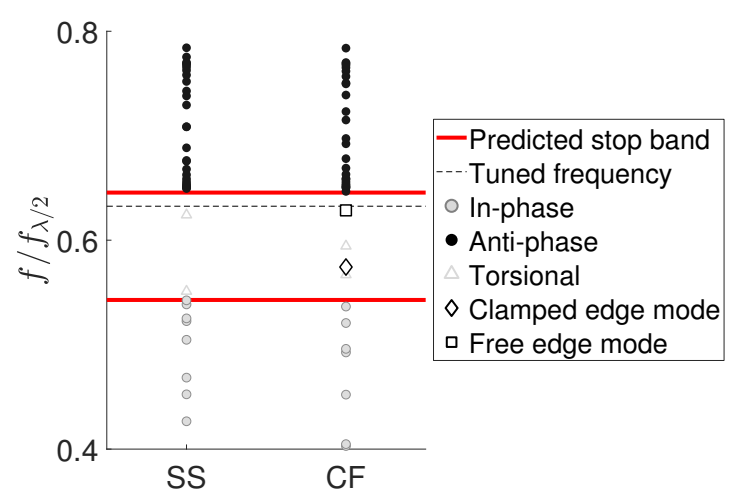

(a)

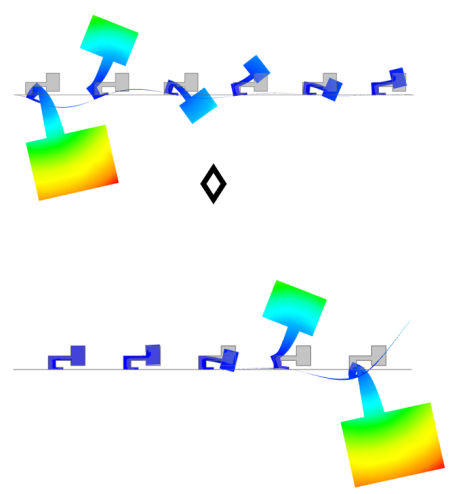

口

(b)

Figure 12: Numerical results from the modal analysis on the SS and CF LRM beam: (a) Natural frequency distribution for the finite LRM beam with SS and CF BCs, (b) mode shapes of the edge modes in the predicted stop band frequency range. Zoom at the clamped side (top) and free side (bottom) of the beam. The undeformed LRM beam is represented by the light grey colour.

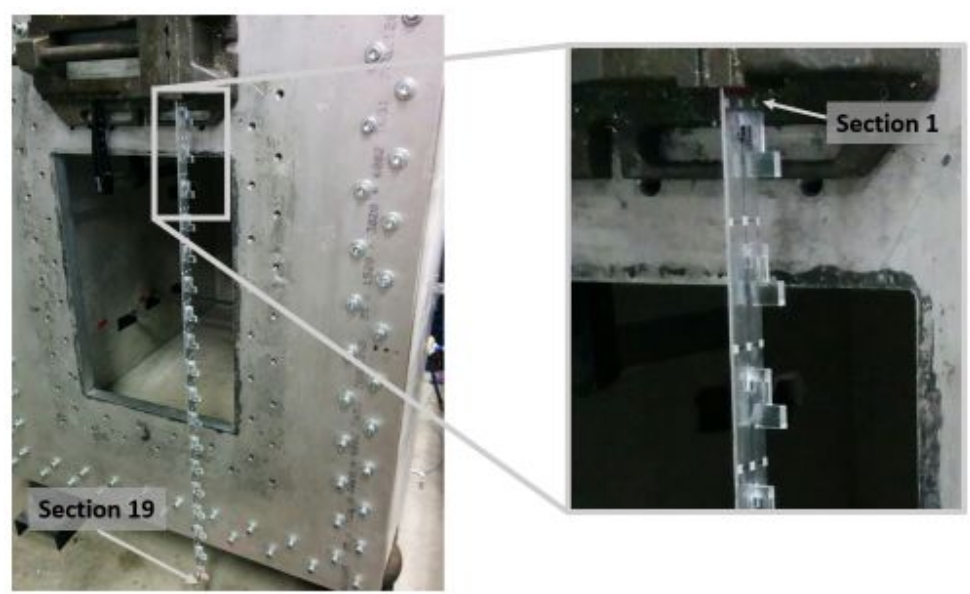

Figure 13: Experimental setup of the CF LRM beam. The first and the last measurement sections are indicated. 

element, which leads to resonant elements with a changed frequency but a comparable mass.

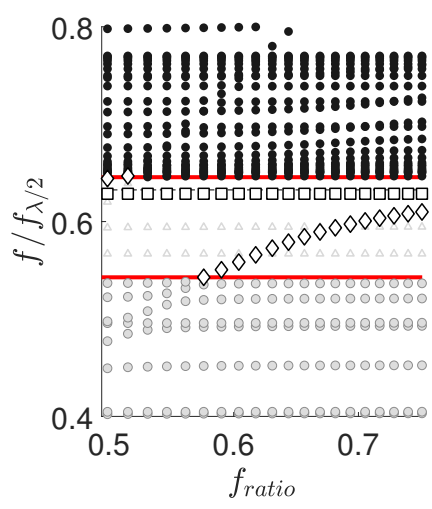

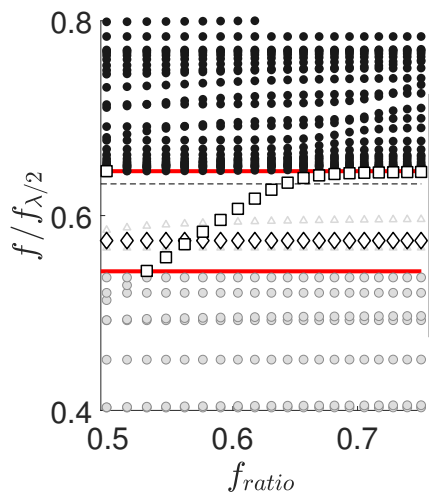

(b) (a)

\begin{tabular}{|l|}
\hline - Predicted stop band \\
-- Tuned frequency \\
- In-phase \\
- Anti-phase \\
Torsional \\
$\diamond$ Clamped edge mode \\
$\square$ Free edge mode \\
\hline
\end{tabular}

Figure 14: Natural frequency distribution of the CF LRM beam with different tuned frequency of the edge resonators. (a) Variation of the tuned frequency for the clamped edge resonator only. (b) Variation of the tuned frequency for the free edge resonator only.

The Young's Modulus is varied between $3 e 9 \mathrm{GPa}$ and $7 e 9 \mathrm{GPa}$ with a step of $0.2 e 9 \mathrm{GPa}$. A modal analysis is run for each configuration. The tuned frequency of only the resonant element at the clamped edge (Figure 14(a)) and at the free edge (Figure 14(b)) is varied.

The clamped edge mode is moved outside the predicted stop band for the resonant element at the clamped edge tuned between $0.515 \leq f_{\text {ratio }} \leq 0.575$. The free edge mode is moved outside the stop band for a tuned 


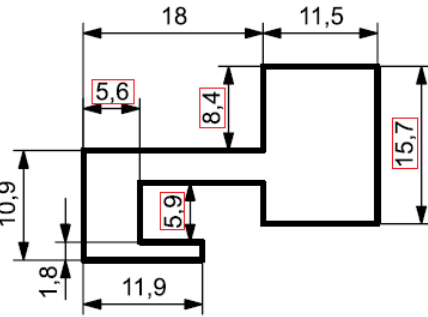

(a)

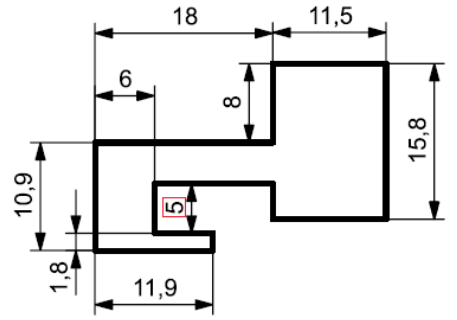

(b)

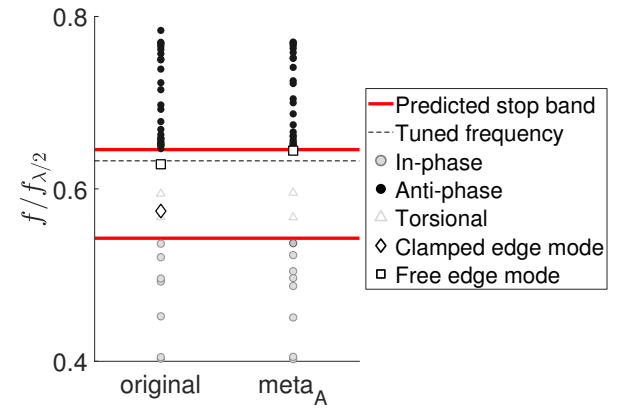

(c)

Figure 15: (a) Dimensions of the clamped edge resonator. (b) Dimensions of the free edge resonator. The dimensions are given in $\mathrm{mm}$. The dimensions which have changed with respect to the original design are indicated with a red box. (c) Comparison of the natural frequency distribution between the original and meta $A$ configuration for the finite LRM beam.

frequency of the resonant element at the free edge between $0.501 \leq f_{\text {ratio }} \leq 0.513$ or is pushed to the upper

In view of minimising the geometrical variation of the current resonant element design, the new resonant elements at the clamped and free edge are tuned at $f_{\text {ratio }}=0.524$ and $f_{\text {ratio }}=0.722$, respectively. The resonant element at the free edge is chosen to be tuned to a higher frequency rather than a lower frequency,

The dimensions of the resonant element at the clamped edge and the free edge of the new LRM beam, called $m e t a_{A}$, is depicted in Figure 15(a) and (b). The geometry of the new resonant elements is obtained by applying small changes to the initial geometry of the resonant element in Figure 10. The relative mass variation with respect to the original resonant element is of $2.6 \%$ and $2 \%$ for the resonant element at the clamped and free edge, respectively. As depicted in Figure 15(c), no clamped edge mode is present anymore the stop band for the meta $_{A}$ configuration and the free edge mode is pushed to the upper stop band bound, in good agreement with the results in Figure 14.

Numerical FRFs are calculated in NX Nastran 12 using SOL108 direct frequency response analysis [45], and are compared to the experimental results in Figure 16 for the original and $m e t a_{A}$ configuration. In the original configuration, the pronounced peaks in the predicted stop band indicate the location of the edge modes, which are well captured by the numerical and experimental results. The small peaks at $f_{\text {ratio }}=0.567$ and $f_{\text {ratio }}=0.594$ are caused by the torsional modes, which are slightly excited during the test. For the excitation at the clamped edge (Figure 16(a)), the edge mode is at a higher frequency as compared to the numerical simulation. This shift can be attributed to the scatter in the resonance frequency of the manufactured resonant elements and the accuracy in the positioning of the resonant element. The edge modes are highly sensitive to both the variation of the tuned frequency and the position from the edge, as will be shown in the following subsections. For the excitation at the free edge (Figure 16(b)), the free edge 
mode is in good agreement with the numerical simulation.

Comparing the original and the $\operatorname{meta}_{A}$ configuration, one can observe that in the latter the modes are moved outside the predicted stop band and the range of attenuation coincides with the designed stop band frequency range. The clamped edge mode shifts below the lower bound of the stop band, whereas the free edge mode moves to the upper limit of the stop band, as predicted. After the upper bound of the stop band, the $m e t a_{A}$ configuration has a higher vibration response than the original configuration, due to the redistribution of the modes in that frequency range.

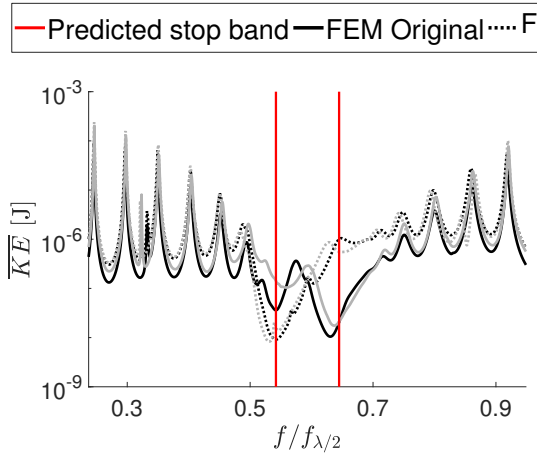

(a)

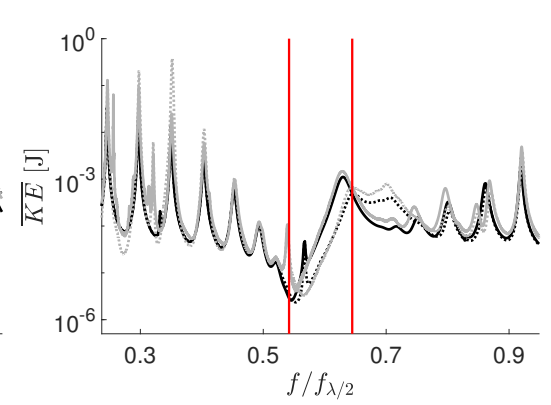

(b)

Figure 16: Comparison of the numerical and experimental $\overline{K E}$ results for the CF LRM beam in original and $m e t a_{A}$ configuration. (a) Excitation in section 1 at the clamped edge. (b) Excitation in section 19 at the free edge.

\subsubsection{Variation of the number of the resonant elements}

Next, the resonant elements are, in consecutive simulations, simultaneously removed from each side of the LRM beam. In Figure 17, it can be observed that no edge modes are present in the predicted stop band when two resonant elements are removed from each side of the beam. This new LRM configuration, named meta $a_{B}$, is chosen for the experimental validation.
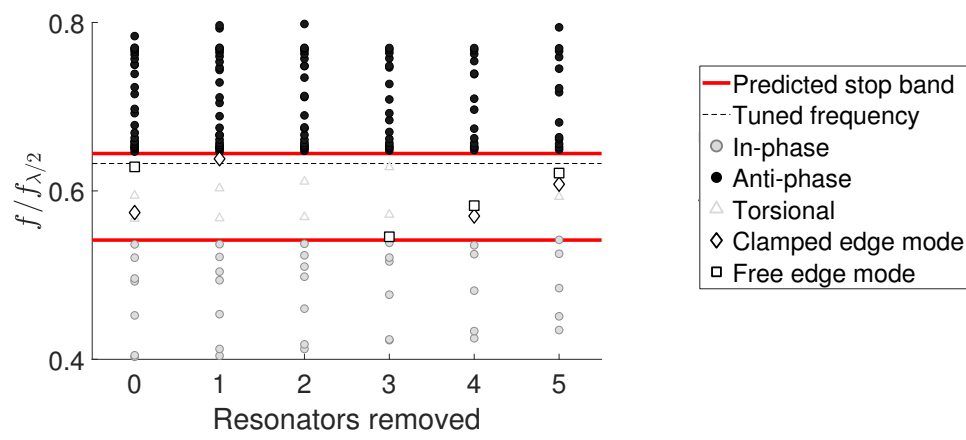

Figure 17: Natural frequencies distribution for the realized CF LRM beam with resonators removed from both the boundaries.

The simulated and experimentally retrieved FRF are given in Figure 18. The peaks in the response of 

predicted stop band for $m e t a_{B}$ configuration is reduced with respect to the original configuration.

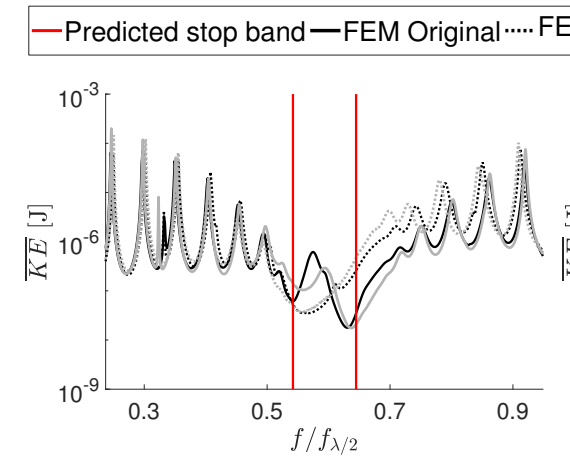

(a)

the original configuration, at $f_{\text {ratio }}=0.524$ for the clamped edge excitation and at $f_{\text {ratio }}=0.722$ for the free edge excitation, are no longer present in the meta $a_{B}$ configuration. Meta $a_{B}$ configuration is about $5 \%$ lighter than the original configuration due to the lower added mass. Given that less resonant elements are present in the LRM beam, less dynamic mass acts on the host structure. As a result, the attenuation in the

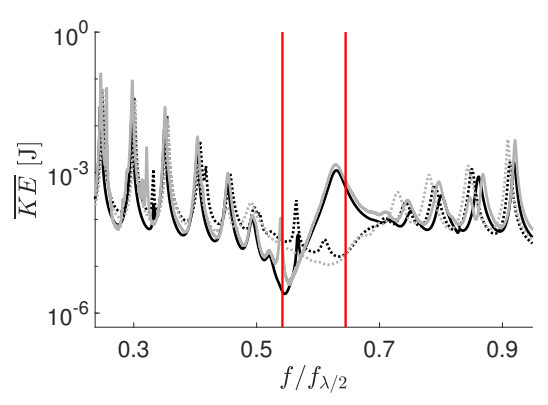

(b)

Figure 18: Comparison of the numerical and experimental $\overline{K E}$ results for the original and $m e t a_{B}$ LRM beam configurations. (a) Excitation in section 1 at the clamped edge. (b) Excitation in section 19 at the free edge.

\subsubsection{Variation of the position of the resonant elements on the beam}

Lastly, the effect of the position of the resonant elements is investigated. All the resonant elements are moved in the positive $\mathrm{x}$-axis direction, according to the reference system in Figure 11, with a step of $2.5 \mathrm{~mm}$. The resonant element position in the UC is indicated by the centre of its base and represented by the red dot in Figure 19(a). As can be seen in Figure 19(b), both the clamped and the free edge modes do not occur anymore in the predicted stop band when the resonant structures are located further from the clamped edge and closer to the free edge, confirming the results of section 4.3.

For the experimental validation, the chosen configuration, named meta $a_{C}$, corresponds to an LRM configuration with the resonant elements at the centre of the UC, located at $30 \mathrm{~mm}$ from the edge of the UC (Figure 19(c)).

The experimental and simulated $\overline{K E}$ are compared in Figure 20. The peaks in the predicted stop band, corresponding to edge modes, are no longer present in configuration met $a_{C}$. The clamped edge mode shifts below the lower bound of the stop band, whereas the free edge mode shifts above the upper bound of the stop band, as predicted.

\subsubsection{Comparison of the test results for the three analysed solutions}

The experimental $\overline{K E}$ results are compared in Figure 21 for configurations meta $a_{A}$ meta $a_{B}$ and $m e t a_{C}$. The vibration response of the original configuration is used as a baseline. 


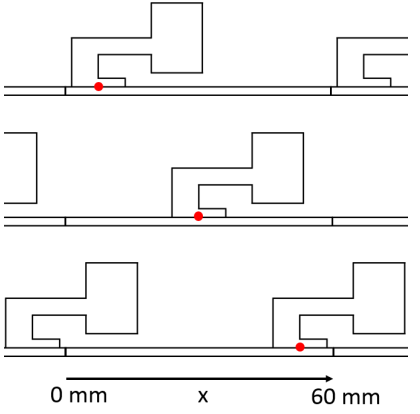

(a)

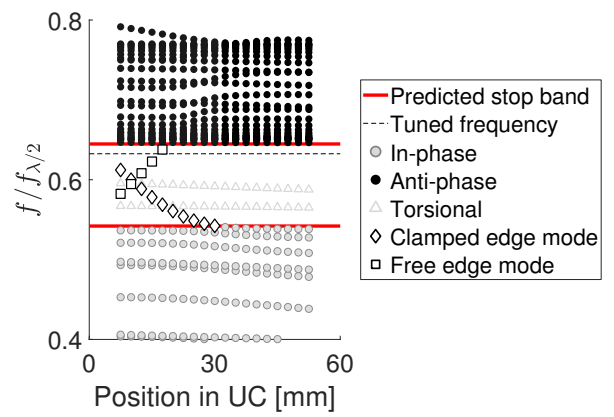

(b)

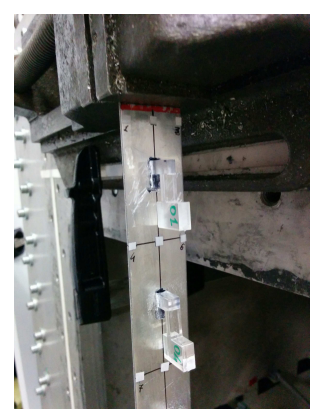

(c)

Figure 19: Variation of the position of the resonant element in the UC for the LRM beam. (a) Sketch of three different positions of the resonant element in the UC. The red dot indicates the centre of the base of the resonant element. (b) Natural frequency distribution of the CF LRM beam with respect to the position of the resonant element in the UC. The position of the resonant element in the UC refers to the red dot in (a). (c) Picture of the LRM beam with resonant elements at 30 mm from the edge of the UC.

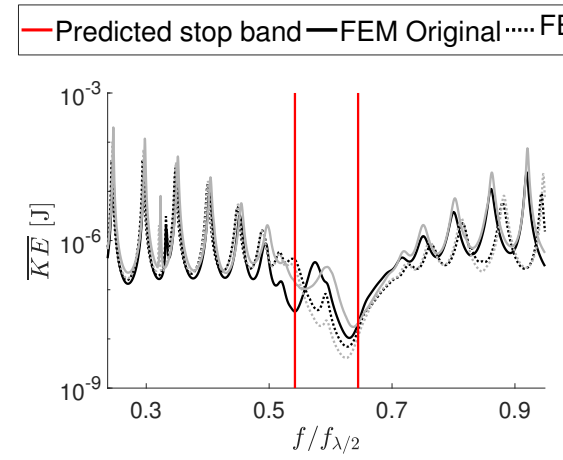

(a)

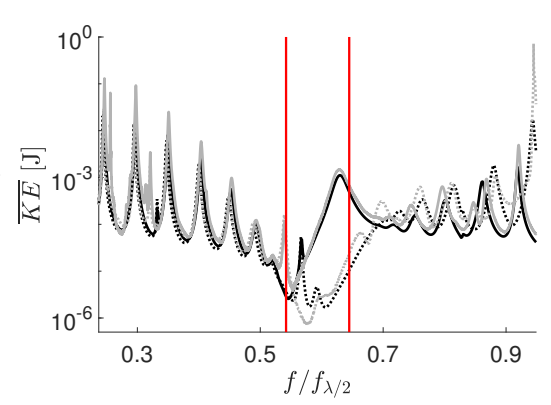

(b)

Figure 20: Comparison of the numerical and experimental $\overline{K E}$ results for the original and meta $a_{C}$ LRM beam configurations. (a) Excitation in section 1 at the clamped edge. (b) Excitation in section 19 at the free edge. 

the modes after the stop band is normally observed [34].

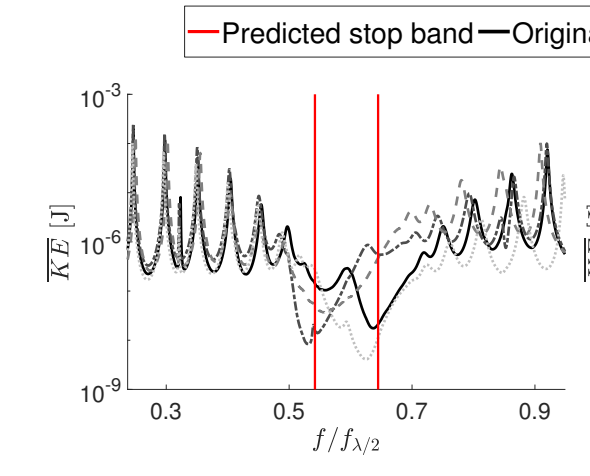

(a) in the stop band.

For all the new configurations, edge modes are not anymore present inside the predicted stop band. However, the vibration attenuation achieved in the stop band is different among the configurations: the way the resonant elements are added to the finite beam strongly influences the vibration attenuation performance achieved in the stop band. Configuration $m e t a_{C}$ achieves the highest vibration attenuation in the predicted stop band. This can be explained by the fact that configuration meta $a_{C}$ has a resonant element in each UC, differently from configuration met $_{B}$, and the same tuned frequency is used for each resonant element on the beam, differently from configuration meta $a_{A}$. As a result, configuration meta $a_{C}$ has a higher dynamic mass acting on the host structure throughout the stop band, resulting in an improvement of the attenuation

The vibration level of configuration $m e t a_{C}$ is also reduced in the frequency range above the upper bound of the stop band. In this frequency range, an increase in the vibration level due to the anti-phase motion of

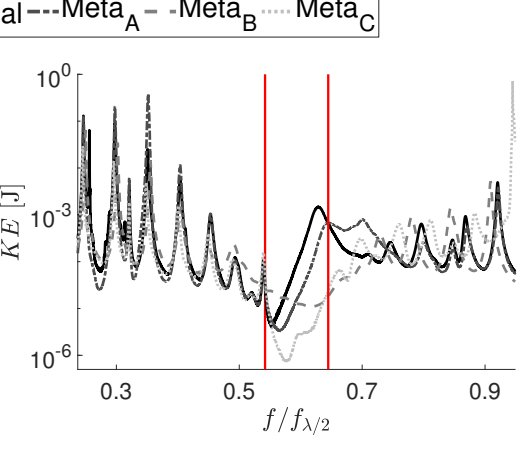

(b)

Figure 21: Comparison of the experimental $\overline{K E}$ results of the original and meta $A_{A}$, meta $a_{B}$ and meta $_{C}$ configurations of the $\mathrm{CF}$ LRM beam. (a) Excitation in section 1 at the clamped edge. (b) Excitation in section 19 at the free edge.

\section{Conclusions}

This paper investigates the effect of BCs on the predicted stop band in a finite LRM beam. Edge modes are present in the predicted stop band in a finite LRM beam and they reduce the level of vibration attenuation achieved. The natural frequency associated with these edge modes is shown to be sensitive to (i) the BCs applied, (ii) the length of the beam, (iii) the added mass and the tuned frequency of the resonant elements on the beam.

In view of controlling the edge modes and preserving the predicted stop band, three solutions are analysed. These solutions consist of varying the local mechanical properties of the LRM beam by varying (i) the tuned frequency, (ii) the number and (iii) the position of the resonant elements in the beam. The effectiveness and the limitation of these solutions are systematically investigated for different configurations of LRM beams 
with different $\mathrm{BCs}$, and the edge modes are shown to be sensitive to the variation of these local mechanical properties.

The experimental results are carried out on different configurations of a LRM beam with CF BCs: the original configuration that has edge modes, and three configuration, according to each of the proposed strategies to control edge modes, resulting in no edge modes. The experimental results are in good agreement with the numerical results.

Finally, the experimental results of the different configurations of the LRM beams are compared. Following, the strategy to control the position of the resonant elements is demonstrated to achieve the highest vibration attenuation inside and outside the stop band frequency range.

\section{Acknowledgements}

The Research Fund KU Leuven is gratefully acknowledged for its support. This research was partially supported by Flanders Make, the Strategic Research Centre for the manufacturing industry. The research of E. Deckers is funded by a grant from the Research Foundation - Flanders (FWO).

\section{References}

[1] L. Fritschi, A. Brown, R. Kim, D. Schwela, S. Kephalopolous, Burden of disease from environmental noise: quantification of healthy life years lost in europe. 2011, Copenhagen: World Health Organization, Regional Office for Europe.

[2] P. K. Mallick, Materials, design and manufacturing for lightweight vehicles, Elsevier, 2010.

[3] F. J. Fahy, P. Gardonio, Sound and structural vibration: radiation, transmission and response, Elsevier, 2007.

[4] L. Brillouin, Wave propagation in periodic structures: electric filters and crystal lattices, Courier Corporation, 2003.

[5] M.-H. Lu, L. Feng, Y.-F. Chen, Phononic crystals and acoustic metamaterials, Materials today 12 (12) (2009) $34-42$.

[6] J. V. Sánchez-Pérez, D. Caballero, R. Mártinez-Sala, C. Rubio, J. Sánchez-Dehesa, F. Meseguer, J. Llinares, F. Gálvez, Sound attenuation by a two-dimensional array of rigid cylinders, Physical Review Letters 80 (24) (1998) 5325.

[7] C. Goffaux, F. Maseri, J. Vasseur, B. Djafari-Rouhani, P. Lambin, Measurements and calculations of the sound attenuation by a phononic band gap structure suitable for an insulating partition application, Applied physics letters 83 (2) (2003) $281-283$

[8] P. A. Deymier, Acoustic metamaterials and phononic crystals, Vol. 173, Springer Science \& Business Media, 2013.

[9] C. Goffaux, J. Sánchez-Dehesa, A. L. Yeyati, P. Lambin, A. Khelif, J. Vasseur, B. Djafari-Rouhani, Evidence of fano-like interference phenomena in locally resonant materials, Physical review letters 88 (22) (2002) 225502.

[10] Z. Liu, X. Zhang, Y. Mao, Y. Zhu, Z. Yang, C. T. Chan, P. Sheng, Locally resonant sonic materials, Science 289 (5485) (2000) 1734-1736.

[11] F. Lemoult, N. Kaina, M. Fink, G. Lerosey, Wave propagation control at the deep subwavelength scale in metamaterials, Nature Physics 9 (1) (2013) 55.

[12] X. Zhou, X. Liu, G. Hu, Elastic metamaterials with local resonances: an overview, Theoretical and Applied Mechanics Letters 2 (4) (2012).

[13] M. I. Hussein, M. J. Leamy, M. Ruzzene, Dynamics of phononic materials and structures: Historical origins, recent progress, and future outlook, Applied Mechanics Reviews 66 (4) (2014) 040802. 
[14] L. Liu, M. I. Hussein, Wave motion in periodic flexural beams and characterization of the transition between bragg scattering and local resonance, Journal of Applied Mechanics 79 (1) (2012) 011003.

[15] P. F. Pai, H. Peng, S. Jiang, Acoustic metamaterial beams based on multi-frequency vibration absorbers, International Journal of Mechanical Sciences 79 (2014) 195-205.

[16] C. Claeys, N. G. R. de Melo Filho, L. Van Belle, E. Deckers, W. Desmet, Design and validation of metamaterials for multiple structural stop bands in waveguides, Extreme Mechanics Letters 12 (2017) 7-22.

[17] A. Nateghi, L. Van Belle, C. Claeys, E. Deckers, B. Pluymers, W. Desmet, Wave propagation in locally resonant cylindrically curved metamaterial panels, International Journal of Mechanical Sciences 127 (2017) 73-90.

[18] J. Mei, G. Ma, M. Yang, Z. Yang, W. Wen, P. Sheng, Dark acoustic metamaterials as super absorbers for low-frequency sound, Nature communications 3 (2012) 756.

[19] C. Claeys, E. Deckers, B. Pluymers, W. Desmet, A lightweight vibro-acoustic metamaterial demonstrator: Numerical and experimental investigation, Mechanical systems and signal processing 70 (2016) 853-880.

[20] L. D'Alessandro, E. Belloni, R. Ardito, A. Corigliano, F. Braghin, Modeling and experimental verification of an ultra-wide bandgap in 3d phononic crystal, Applied Physics Letters 109 (22) (2016) 221907.

[21] M. Collet, M. Ouisse, M. Ruzzene, M. Ichchou, Floquet-bloch decomposition for the computation of dispersion of twodimensional periodic, damped mechanical systems, International Journal of Solids and Structures 48 (20) (2011) $2837-2848$.

[22] L. Van Belle, C. Claeys, E. Deckers, W. Desmet, On the impact of damping on the dispersion curves of a locally resonant metamaterial: Modelling and experimental validation, Journal of Sound and Vibration 409 (2017) 1-23.

[23] A. Hvatov, S. Sorokin, Free vibrations of finite periodic structures in pass-and stop-bands of the counterpart infinite waveguides, Journal of Sound and Vibration 347 (2015) 200-217.

[24] K. Billon, M. Ouisse, M. Collet, E. Sadoulet-Reboul, Design of smart metamaterials for vibration control: extension of bloch approach to handle finite system boundary conditions, in: Health Monitoring of Structural and Biological Systems XII, Vol. 10600, International Society for Optics and Photonics, 2018, p. 106001J.

[25] R. K. Pal, M. Ruzzene, Edge waves in plates with resonators: an elastic analogue of the quantum valley hall effect, New Journal of Physics 19 (2) (2017) 025001.

[26] B. L. Davis, A. S. Tomchek, E. A. Flores, L. Liu, M. I. Hussein, Analysis of periodicity termination in phononic crystals, in: ASME 2011 International Mechanical Engineering Congress and Exposition, American Society of Mechanical Engineers, 2011, pp. 973-977.

[27] Y. Xiao, J. Wen, G. Wang, X. Wen, Theoretical and experimental study of locally resonant and bragg band gaps in flexural beams carrying periodic arrays of beam-like resonators, Journal of Vibration and Acoustics 135 (4) (2013) 041006.

[28] P. Méresse, C. Audoly, C. Croënne, A.-C. Hladky-Hennion, Acoustic coatings for maritime systems applications using resonant phenomena, Comptes Rendus Mecanique 343 (12) (2015) 645-655.

[29] D. Mead, Wave propagation in continuous periodic structures: research contributions from southampton, 1964-1995, Journal of sound and vibration 190 (3) (1996) 495-524.

[30] B. R. Mace, D. Duhamel, M. J. Brennan, L. Hinke, Finite element prediction of wave motion in structural waveguides, The Journal of the Acoustical Society of America 117 (5) (2005) 2835-2843.

[31] A. Diaz, A. Haddow, L. Ma, Design of band-gap grid structures, Structural and Multidisciplinary Optimization 29 (6) (2005) 418-431.

[32] B. R. Mace, E. Manconi, Modelling wave propagation in two-dimensional structures using finite element analysis, Journal of Sound and Vibration 318 (4-5) (2008) 884-902.

[33] D. J. Mead, A new method of analyzing wave propagation in periodic structures; applications to periodic timoshenko beams and stiffened plates, Journal of Sound and Vibration 104 (1) (1986) 9-27.

[34] C. C. Claeys, K. Vergote, P. Sas, W. Desmet, On the potential of tuned resonators to obtain low-frequency vibrational 
stop bands in periodic panels, Journal of Sound and Vibration 332 (6) (2013) 1418-1436.

[35] G. S. Gupta, Natural flexural waves and the normal modes of periodically-supported beams and plates, Journal of Sound and Vibration 13 (1) (1970) 89-101.

[36] D. J. Mead, Free wave propagation in periodically supported, infinite beams, Journal of Sound and Vibration 11 (2) (1970) 181-197.

[37] W. Weaver Jr, S. P. Timoshenko, D. H. Young, Vibration problems in engineering, John Wiley \& Sons, 1990.

[38] D. Mead, S. Parthan, Free wave propagation in two-dimensional periodic plates, Journal of Sound and Vibration 64 (3) (1979) 325-348.

[39] C. C. Claeys, P. Sas, W. Desmet, On the acoustic radiation efficiency of local resonance based stop band materials, Journal of Sound and Vibration 333 (14) (2014) 3203-3213.

[40] L. Cremer, M. Heckl, Structure-borne sound: structural vibrations and sound radiation at audio frequencies, Springer Science \& Business Media, 2013.

[41] C. Sugino, S. Leadenham, M. Ruzzene, A. Erturk, On the mechanism of bandgap formation in locally resonant finite elastic metamaterials, Journal of Applied Physics 120 (13) (2016) 134501.

[42] Y. Xiao, B. R. Mace, J. Wen, X. Wen, Formation and coupling of band gaps in a locally resonant elastic system comprising a string with attached resonators, Physics Letters A 375 (12) (2011) 1485-1491. doi:10.1016/j.physleta.2011.02.044.

[43] The MathWorks, Inc., Natick, Massachusetts, United States, MATLAB 2018a documentation, 2018th Edition.

[44] L. Sangiuliano, E. Deckers, C. Claeys, Control of edge modes in finite vibro-acoustic resonant metamaterials, in: Proceedings of the 28th International Conference on Noise and Vibration Engineering (ISMA 2018), 2018.

[45] Siemens, NX Nastran 12, Quick Reference Guide, Siemens PLM Software Inc. (2017). 